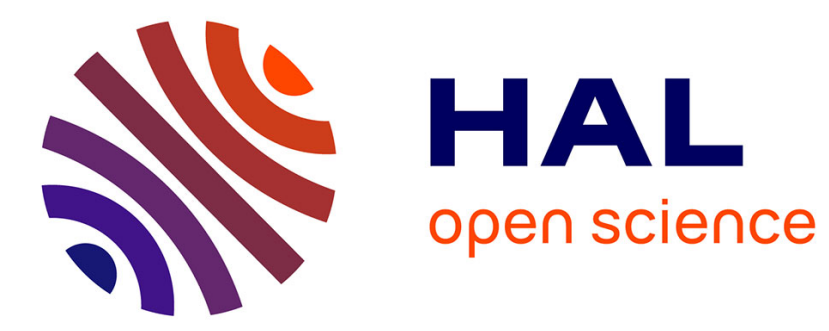

\title{
Retirés et connectés, les hikikomori et les écrans.
}

Natacha Vellut

\section{To cite this version:}

Natacha Vellut. Retirés et connectés, les hikikomori et les écrans.. Revue de l'enfance et de l'adolescence RAFEF-GRAPE, 2017. hal-01984836

\section{HAL Id: hal-01984836 https://hal.science/hal-01984836}

Submitted on 17 Jan 2019

HAL is a multi-disciplinary open access archive for the deposit and dissemination of scientific research documents, whether they are published or not. The documents may come from teaching and research institutions in France or abroad, or from public or private research centers.
L'archive ouverte pluridisciplinaire HAL, est destinée au dépôt et à la diffusion de documents scientifiques de niveau recherche, publiés ou non, émanant des établissements d'enseignement et de recherche français ou étrangers, des laboratoires publics ou privés. 


\section{Revue de l'enfance et de l'adolescence}

« Les défis du virtuel »

Natacha Vellut

Retirés et connectés, les hikikomori et les écrans

\section{Résumé}

A partir d'une analyse d'entretiens avec des jeunes en retrait social (hikikomori) l'auteur cherche à saisir l'articulation entre la réclusion à domicile et les usages et connexions numériques permises par les écrans. Elle souligne la diversité de ces usages, diversité qui est la norme pour les usagers de ces nouvelles technologies. Elle met l'accent sur quatre motivations des jeunes en retrait pour investir ces pratiques: la possibilité de l'expression d'émotions négatives sans crainte et sans jugement, le soutien par des instances imaginaires idéales qui échappent à toute sanction familiale ou sociale, l'accès à un savoir sans passer par un maître, la création d'un espace-temps très spécifique, un temps suspendu qui s'abstient des contraintes du temps et des espaces communs, qui s'abstient de la rencontre réelle, physique, corporelle, avec d'autres.

\section{Mots clés}

Retrait social, réclusion à domicile, usages numériques, Internet, Moi idéal

\section{Introduction}

Ils nous intriguent, nous questionnent. A quoi peuvent-ils passer leurs journées et leurs nuits puisqu'ils ne vont plus à leur collège, lycée ou université, ne voient plus leurs amis, sortent rarement du domicile familial ? "Ils", ce sont ces jeunes épinglés d'un terme japonais : hikikomori. Ce mot composé de hiku (hiki) que nous pourrions traduire par "reculer», et komoru (komori) "se cloîtrer ", apparaît au Japon à la fin des années 1980 et désigne des cas de réclusion à domicile de jeunes, adolescents ou jeunes adultes ${ }^{1}$. Cette réclusion est souvent précédée d'absentéisme scolaire (futōkō). Ces jeunes gens, vivant retirés dans leur chambre, s'abstiennent de toute relation que nous pourrions attendre d'eux à leur âge: ils ne s'investissent plus dans leurs études, ni dans leurs activités de loisir, ni dans des relations amicales ou amoureuses. Ils s'enferment, même si leur réclusion peut être relative, à leur domicile, c'est-à-dire le plus souvent au domicile familial, et se replient sur des relations à minima avec les membres de leur entourage vivant sous le même toit. Quelques rares cas présentent un repli extrême, s'isolent complètement dans leur chambre, ne parlant plus à personne, mangeant ce que leur mère dépose au pied de leur porte. Le phénomène japonais est étudié, analysé et de multiples causalités, qu'elles soient sociales, économiques ou culturelles, lui sont supposées. Une forme de liens sociaux japonais, basée sur une co-dépendance

\footnotetext{
${ }^{1}$ Pour une introduction plus documentée : FANSTEN M., FIGUEIREDO C., PIONNE-DAX N., VELLUT N. (dir.) 2014, Hikikomori, ces adolescents en retrait, Paris, Armand Colin.
} 
mutuelle, exemplifiée dans le lien de la mère à ses enfants, des changements sociétaux trop soudains après la seconde Guerre Mondiale, puis après la grave crise économique du mitan des années 1980, la pression scolaire d'un système éducatif élitiste sont autant de facteurs qui peuvent expliquer l'ampleur de cette conduite au pays du soleil levant. Ailleurs dans le monde, des scientifiques, quelques sociologues et des psychiatres, cherchent à repérer si ce même phénomène existe. En comparaison du Japon où il prend des proportions alarmantes, devient un problème de santé publique et concerne des centaines de milliers de jeunes, le phénomène est discret. Des cas de hikikomori sont néanmoins reportés dans la littérature, principalement psychiatrique, aux Etats-Unis, en Corée du Sud et en Australie, à Oman, à Hong-Kong, en Espagne et en Italie ${ }^{2}$. Une enquête réalisée à partir d'un questionnaire diffusé auprès de psychiatres locaux soutient qu'il existe des cas de hikikomori dans huit pays investigués en dehors du Japon ${ }^{3}$. II n'existe cependant pas de terminologie spécifique, anglaise, italienne, française ou autre, pour nommer ce phénomène. En France, le phénomène et le terme hikikomori commencent doucement à être connus. Comme il n'existe pas d'appellation locale spécifique pour appréhender et qualifier cette réclusion à domicile, elle est repérée à travers d'autres catégories, souvent sous l'angle du décrochage scolaire, ou avec les catégories cliniques de phobie scolaire ou de troubles anxieux. En termes psychopathologiques, le hikikomori est appréhendé de façon contradictoire ${ }^{4}$. Un hikikomori primaire est distingué d'un hikikomori secondaire. Dans le cas du hikikomori primaire, aucun diagnostic ne rend compte du phénomène de retrait social, qui est donc conçu comme primaire et non secondaire à une pathologie psychiatrique ou psychologique, même si ce retrait peut, à son tour, entraîner des pathologies du sommeil, de l'alimentation, des humeurs... Tandis que le hikikomori secondaire pourrait s'expliquer

\footnotetext{
2 TEO, AR. 2012, "Social isolation associated with depression: a case report of hikikomori", The International Journal of Social Psychiatry, 59(4), p.339-41. KIM J, RAPEE RM, OH KJ, MOON H-S. 2008, "Retrospective report of social withdrawal during adolescence and current maladjustment in young adulthood: Cross-cultural comparisons between Australian and South Korean students". Journal of Adolescence, 31(5), p. 543-63. SAKAMOTO N, MARTIN RG, KUMANO H, KUBOKI T, Al-ADAWI S. 2005. "Hikikomori, Is It A Culture-Reactive Or Culture-Bound Syndrome? Nidotherapy And A Clinical Vignette from Oman", International Journal of Psychiatry in Medicine, 35(2), p.191-8. WONG V. 2006, "Social Withdrawal of Young People in Hong Kong: A Social Exclusion Perspective", The Hong Kong Journal of Social Work (HKJSW), 40(1-2), p. 61-91. GARCIA-CAMPAYO J, ALDA M, SODRADIEL N, SANZ ABOS B. 2007, "A Case Report of Hikikomori in Spain”, Medicina Clínica, 129(8), pp.318-9. OVEJERO S., CARO-CANISARES I., DE LEON-MARTINEZ V., BACA-GRACIA E. (2013). « Prolonged social withdrawal disorder. A hikikomori case in Spain ", International Journal of Social Psychiatry, 6. SAGLIOCCO G. (dir.). 2011, Hikikomori e adolescenza. Fenomenologia dell'autoreclusione [Hikikomori and Adolescence. Phenomenology of Self-Seclusion]. Milano-Udine, Mimesis.

${ }^{3}$ L'Australie, le Bangladesh, l'Inde, I'Iran, la Corée du Sud, Taiwan, la Thaïlande et les Etats-Unis sont les huit pays dans lesquels les questionnaires ont été envoyés. KATO T., MASARU T., SHINFUKO N., FUJISAWA D., TEO A.-R., SARTORIUS N., AKIYAMA T. et al. (2012). " Does the "Hikikomori" Syndrome of Social Withdrawal Exist Outside Japan? A Preliminary International Investigation. " Social Psychiatry and Psychiatric Epidemiology, 477, 1061-75

${ }^{4}$ GARIUP M, PARELLADA E, GARCIA C, BERNARDO M. 2008, "Hikikomori or Simple Schizophrenia?", Medicina Clinica,130(18), pp.718-9. HIRASHIMA N. (2001). "Psychopathology of Social Withdrawal in Japan ", Journal of the Japan Medical Association, 44-6, 260-262. KOYAMA A., MIYAKE Y., KAWAKAMI N., TSUCHIYA M., TACHIMORI H. \& TAKESHIMA T. (2010). "Lifetime prevalence, psychiatric comorbidity and demographic correlates of "hikikomori" in a community population in Japan ". Psychiatry research, 176-1, 69-74. MALAGON A., ALVARO P., CORCOLES D., MARTIN-LOPEZ L.M., BULBENA M., (2010), " Hikikomori': a New Diagnosis or a Syndrome Associated with a Psychiatric Diagnosis? ", The International Journal of Social Psychiatry, 56-5, 558559. SUWA M. \& SUZUKi K. (2002). "Psychopathological features of a "primary social withdrawal" ». Seishin Shinkeigaku Zasshi, 104, 1228-1241. (en japonais). SUWA, M. \& SUZUKI, K. (2013). The phenomenon of "hikikomori" (social withdrawal) and the socio-cultural situation in Japan today. Journal of Psychopathology, 19, 191-198. Voir aussi KATO et al. (2012), GARCIA-CAMPAYO et al. (2007).
} 
par une pathologie antérieure. Tadaaki Furushahi et Natacha Vellut soulignent la diversité ou l'absence de diagnostics associés au retrait dans leur étude de 20 cas de retrait, 10 français et 10 japonais $^{5}$. Quand il s'agit de qualifier le phénomène en pathologie, les diagnostics oscillent entre culture-bound syndrome, un syndrome lié à la culture, typiquement japonais ${ }^{6}$, nouveau trouble psychiatrique, donc primaire, et toute une série de troubles déjà répertoriés: troubles anxieux, dépressifs, phobiques, du développement, de la personnalité comme pour Garcia-Campayo et al. (2007) qui l'associent aux troubles de la personnalité paranoïaque. La réclusion à domicile est, enfin, souvent associée à un usage excessif des jeux vidéo, nous y reviendrons.

L'ampleur du champ et du questionnement diagnostiques laisse à penser qu'il est finalement difficile d'associer le hikikomori à un trouble spécifique. Il s'agit d'une conduite dont, somme toute, nous savons peu de choses, ni ce qu'elle recouvre, ni ce qu'elle exprime, et jusqu'à ce qui la constitue au quotidien qui nous échappe puisque ces jeunes s'extraient de tout regard social. Certains scientifiques, y compris cliniciens, défendent une interprétation qui dé-pathologise ce comportement, le pensent comme un compromis ou une solution pour faire face aux difficultés du passage vers le monde adulte, comme une possibilité de questionnement, réaménagement, réajustement de ses choix et investissements. Pour la psychologue Tanaka, le retrait social est ainsi temporaire et utile et permet au jeune de repenser sa vie et réaliser un travail sur soi. ${ }^{7}$

\section{Hikikomori et usages numériques}

Comme écrit précédemment, le hikikomori est souvent associé à un usage abusif voire addictif aux jeux vidéos. C'est une opinion communément partagée dans les médias, la littérature scientifique ou l'opinion publique qui s'appuie sur un parallèle entre la diffusion d'internet, particulièrement vivace au Japon, et le développement de cette réclusion à domicile des jeunes. Le Japon est un pays fervent de nouvelles technologies de l'information qui y représentent le premier secteur économique, marché très innovant soutenu par des programmes gouvernementaux. Le Japon est ainsi classé au premier rang mondial pour l'accès à internet sur mobile et l'accès à internet y est le moins cher au monde selon l'ITU $^{8}$. En ce qui concerne les jeux vidéo, le marché japonais est quantitativement aussi important que le marché américain.

Ce graphique (en japonais) compare la diffusion des NTIC (en abscisse) et la publication d'articles sur le hikikomori (en ordonnée) au Japon? .

\footnotetext{
${ }^{5}$ FURUHASHI T, VELLUT N. Si proches, si lointains : hikikomori en France et au Japon. In: Fansten M, Figueiredo C, Pionné-Dax N, Vellut N, (dir.) 2014, Hikikomori, ces adolescents en retrait. Paris: Armand Colin, p. 139-56.

${ }^{6}$ TEO, A. R \& GAW C. (2010). "Hikikomori, a Japanese culture-bound syndrome of social withdrawal? A proposal for DSM-5 », The Journal of nervous and mental disease, 198-6, 444-449

${ }^{7}$ HORIGUCHI S. (2011). " Hikikomori. How private isolation caught the public eye », in Goodman R., Imoto Y. \& Toivonen T. (dir.), A Sociology of Japanese Youth: From Returnees to NEETs, Abington, Routledge, 122-138

${ }^{8}$ L'Union internationale des télécommunications (UIT, ou en anglais International Telecommunication Union, ITU) est l'agence des Nations Unies pour le développement spécialisée dans les technologies de l'information et de la communication. Elle est basée à Genève (Suisse).

${ }^{9}$ Graphique présenté par le Dc Kunifumi Suzuki, psychiatre à l'Université de Nagoya, lors d'un colloque en 2010 au Centre professionnel et de pédagogie appliquée (CPPA) du Département du Val-de-Marne.
} 


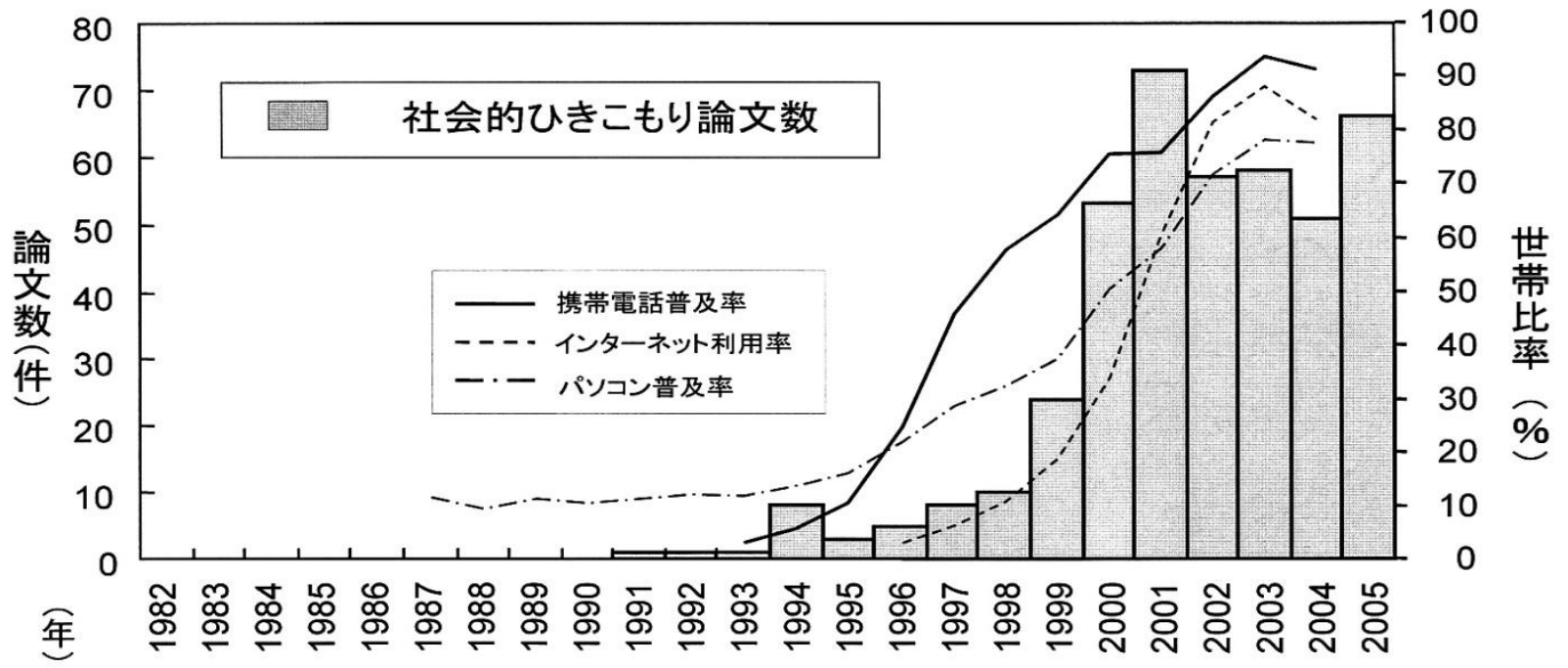

téléphone mobile

internet

---_ ordinateur

Pouvons-nous simplement conclure qu'il s'agit d'une coïncidence temporelle et territoriale ? D'autant que certains portraits de joueurs vidéo excessifs ressemblent de façon troublante aux jeunes hikikomori : ce sont majoritairement des jeunes hommes, âgés de 14 à 25 ou 30 ans, souvent déscolarisés ou inactifs comme le souligne Mickael Stora ${ }^{10}$, qui se présentent volontiers comme de bons élèves avant leur décrochage scolaire, apparaissant comme timides, peu à l'aise dans les relations sociales, en particulier les relations avec leurs pairs. Cependant, si nous étudions finement la vingtaine d'entretiens de recherche que nous avons mené avec de jeunes hikikomori en France et au Japon ${ }^{11}$, le jeu excessif n'apparaît qu'une seule fois comme causal du retrait à domicile. C'est l'explication donnée en première instance par le jeune lui-même, explication qui pourrait bien sûr en cacher une autre que seul un suivi thérapeutique dans la durée pourrait mettre à jour.

Laurent, 17 ans, qui vient de sortir de 4 ans de retrait témoigne :

Je suis tombé addict à 13 ans sur le jeu Dofus. Ça a duré près de deux ans. Je sortais plus, j'allais plus en cours, etc. Je commençais à être déscolarisé. Après ça a suivi [le retrait social] on s'accroche au personnage et on veut le faire monter au maximum, du coup le temps il passe. [...] Je voulais que faire ça. Je voulais tout le temps jouer.

L'analyse de l'emploi du temps des jeunes en retrait est difficile à mener compte tenu de leur rapport au temps très particulier, le temps en effet " passe " sans que rien ne semble faire repère signifiant. Les jeunes peuvent ainsi décrire des journées entières d'un simple "j'ai surfé sur internet ", "j'étais dans ma chambre ", "j'ai joué ». Si nous additionnons les heures passées devant un écran, quel qu'il soit : télévision, ordinateur, tablette, smartphone, pour y faire quoi que ce soit, regarder un film, une série, s'informer, jouer, apprendre, etc., des jeunes en retrait cumulent plus de 10 heures quotidiennes devant leurs écrans. Il semble donc important de s'y intéresser spécifiquement et nous

\footnotetext{
${ }^{10}$ STORA, M. 2008, "Tout objet de plaisir peut devenir une addiction ", La santé de l’homme, n³96, p.4-6

${ }^{11}$ Grâce à une équipe de recherche dont le « noyau dur » est constitué de l'auteur, de Maïa Fansten, sociologue à I'Université Paris Descartes, Cristina Figuereido, anthropologue à I'Université Paris Descartes, et Tadaaki Furuhashi, psychiatre à l'Université de Nagoya.
} 
menons plusieurs entretiens avec des jeunes retirés ou sortis de leur retrait sur ce thème exclusif. L'analyse des entretiens biographiques de recherche et de ces longs entretiens ciblés produit un tableau riche, nuancé, complexe et finalement très ambivalent des usages numériques des jeunes en retrait social. Cette analyse nous permet d'un peu mieux comprendre quels sont ces usages et quelles sont leurs effets à la fois sur la conduite de retrait et sur le jeune lui-même.

En premier lieu, l'étude atteste de l'extrême variété des usages du numérique chez les jeunes en retrait, ce qui ne les distingue pas des autres usagers du numérique. La variété de ces usages est documentée dans la littérature scientifique. Il est par exemple décrit que sur Facebook les internautes peuvent partager et écouter de la musique, jouer sur des applications, regarder les photos ou les vidéos de leurs amis, suivre les actualités politiques et/ou sportives, suivre l'actualité Facebook en mode hors-ligne, discuter avec des amis, etc. Etre en lien online ou se chercher de nouveaux amis sont donc loin d'être les seules activités d'un réseau social. ${ }^{12}$ Les outils de communication sur Internet sont multiples, la diversité des applications est vertigineuse, comme les motivations pour se connecter. Ainsi, les raisons de jouer à des jeux vidéo sont diverses : plaisir de la compétition, régulation émotionnelle (oubli de ses problèmes, barrage contre sa solitude, etc.), possibilité de se constituer un réseau d'amis, apprentissage entre pairs, opportunité de développer des compétences techniques comme relationnelles (médiation, persuasion, leadership, etc.), recherche de la sensation du flow (être absorbé), expression de sa créativité, expérimentation de plusieurs identités, découverte de nouveaux environnements et apprentissage de nouvelles techniques de jeu, etc. La longue interview par Serge Tisseron d'Elie Rotenberg ${ }^{13}$, qui a dirigé une des meilleures guildes françaises pour le jeu en réseau World of Warcraft, témoigne que les joueurs de jeux numériques acquièrent des compétences et un esprit de performance qui plaisent à beaucoup d'entreprises et leur ouvrent des opportunités professionnelles. Rien, donc, qui prédestinerait au retrait social.

Quelles sont les motivations des hikikomori à s'installer devant leurs écrans, à se connecter à internet ? Nous en distinguerons quatre dans le cadre de cet article, sans prétendre à l'exhaustivité, et nous tenterons de préciser leur articulation avec la conduite de retrait.

\section{Exprimer sa haine, sa peur, sa colère}

L'adolescence se caractérise par le développement de l'excitation érotique, la puberté et sa fonction de réveil pulsionnel, et un engagement dans la recherche de son identité qui peut discréditer les instances parentales. Ainsi advient une énergie qui peut déborder le jeune et s'avérer destructrice. L'adolescence est ce moment de conflagration pour l'énergie sexuelle et pulsionnelle qui se présente en trop, en excédent. Cette énergie, comme le remarque Winnicott ${ }^{14}$, est à comprendre au sens large. Il ne s'agit pas uniquement des moyens physiques et libidinaux d'atteindre et de jouir de l'objet sexuel mais aussi des moyens physiques et libidinaux de détruire. "La problématique adolescente se déploie autour du statut et de la fonction de l'interdit, et c'est de là que procède le rapport à l'objet. L'enjeu n'est pas de 'tuer le père', comme on entend le dire, mais de supporter qu'il soit mort, comme instance au-delà du père imaginaire. ${ }^{15}$ La pulsion de mort est donc particulièrement présente à l'adolescence et il s'agit pour le jeune de s'y affronter. Cette pulsion est, à notre époque relativement pacifiée, peu prise en compte dans le social qui ne comporte actuellement en France, ni guerre, ni conflit ouvert, ni service militaire. L'adolescent peut se sentir seul à se confronter à cette pulsion et il demande à ses pairs, au groupe, offline ou online, de l'aider à prendre en charge cette énergie

\footnotetext{
12 NASR, HE et SAIED BEN RACHED, K. 2015, « La cyberdépendance : cas de l'addiction au réseau social Facebook ", La revue gestion et organisation, 2015/7, p. 125-134

${ }^{13}$ TISSERON, Serge. 2012, "Interview d’Elie Rotenberg », Adolescence, 2012/1, n79, p.63-87.

${ }^{14}$ WINNICOTT, DW. L'adolescence (1962) in The Family and individual Development, 1965, Londres, Tavistock.

${ }^{15}$ VANIER, Alain. 2001, "Some remarks on Adolescence with Particular Reference to Winnicott and Lacan", The Psychoanalytic Quartely, volume LXX, n³, New York, July 2001, pp.579-597
} 
destructrice. Les relations online se caractérisent « de ne pas s'inscrire dans le système hiérarchique de la société mais de se produire entre individus occupant la même position sociale [...] le désir de parler s'exerce en dehors des cadres contraignants de la société. ${ }^{16}$ Les relations online forment un groupe de pairs et la socialisation via Internet permet de s'associer à des semblables. Ces semblables que le jeune adulte doit rejoindre pour assumer une position sexuée, une position déterminée dans la sexuation. Semblables d'autant plus semblables que le jeune n'en a qu'une connaissance très partielle. Il peut donc les imaginer semblables sur la seule base d'un même flux de communication et d'un même langage (sur les forums se développent des langages spécifiques, compréhensibles uniquement par les initiés). Les relations, voire les amitiés, online permettent plus facilement l'expression hostile, l'exposition de soi, ouvrent à plus de sujets de discussions, sans crainte du jugement, d'autant qu'on peut s'y présenter anonymement. ${ }^{17}$ Internet produit un effet de désinhibition étudié par le psychologue américain John Suler. Nous donnons ici l'exemple d'un textboard japonais, 2 channel $^{18}$, fréquemment consulté par Katsushi, japonais de 21 ans, en retrait depuis 2 ans. 2 channel a, dans la société japonaise, une influence comparable à celui de mass-médias traditionnels comme la télévision, la radio ou les journaux. Une de ses caractéristiques distinctives est la liberté complète et anonyme des messages postés, a contrario de la plupart des forums Internet qui exigent une forme d'inscription, souvent une identification de l'usager par son adresse de courrier électronique. Le fondateur de 2 channel, Hiroyuki Nishimura, "lisant l'air ${ }^{19}$ " explique qu'il s'est rendu compte qu'au Japon un espace d'expression sans entrave était nécessaire. II a donc créé ce forum sur lequel il n'y a aucun censeur, aucun filtre, aucune vérification d'âge, aucun système de vote qui stimule un fil de discussion. "J'ai créé un espace de liberté et ce que les gens en font leur appartient. [Les utilisateurs] peuvent discuter, se disputer, se plaindre, insulter, imaginer, associer librement, plaisanter, se distraire, se délecter de leur pouvoir de considérer l'autre comme un parfait anonyme. ${ }^{20}$

Ludovic, jeune français de 16 ans, depuis 2 ans en retrait, en témoigne aussi :

" Je vais sur des sites où on est complètement anonyme, où on n'a pas de trace de ce qu'on écrit. Et même là [au début de son retrait] j'arrivais pas à discuter, même en anonyme. J'avais peur d'être ignoré... [...] J'étais sur un site assez hostile quand même où il n'y a pas de modération [4chan]. C'est un peu une baston en permanence. C'est l'endroit où j'ai les meilleures discussions possibles parce qu'il y a pas de modération. Les gens ne s'inquiètent pas de ce qu'ils disent, donc il y a un peu de tout. Tu peux avoir de très, très bonnes conversations comme des trucs complètement débiles. [...] Une liberté d'expression totale ${ }^{21}$. Il y a des gens qui vous disent que c'est un endroit absolument atroce.

\footnotetext{
${ }^{16}$ BRIOLE A. et TYAR A. 1987, Fragments de passions ordinaires. Essai sur le phénomène de télésociabilité, Paris, La documentation française. Cette étude montre que la télé-convivialité expérimentée par téléphone préfigure les phénomènes de rencontre à distance qui se développeront via les messageries roses du minitel.

${ }^{17}$ WONG, V. and YING, W. Young people and social withdrawal : a social exclusion perspective. Paper presented at $7^{\text {th }}$ International Conference on Asian Youth Issues : Opportunities and Challenges of Youth in New Era, Macao Special Administration Region, China

${ }^{18}$ Le nom "2channel" serait une référence aux anciens joueurs vidéo qui connectaient leur console de jeu via le canal 2 de la bande VHF du poste de télévision familial.

${ }^{19}$ L'expression japonaise « lire l'air » signifie qu'il faut savoir sentir l'atmosphère d'une situation, qu'il faut savoir comprendre à la fois le non et le non-dit d'une personne, d'un groupe, décrypter une gêne, une demande non formulée.

${ }^{20}$ Sur 2channel, compte tenu de sa taille et de l'anonymat, la calomnie, le discours de haine et la diffamation contre des figures publiques, des institutions, des minorités et des groupes ethniques spécifiques sont courants. Les discussions peuvent déboucher sur des démonstrations réelles comme, par exemple, un rassemblement en août 2011 contre la Télévision Fuji sous prétexte que la chaîne diffusait trop d'émissions de télévision coréennes.

${ }^{21}$ Ludovic serait en ce sens un libertarien, quelqu'un pour qui la liberté d'expression est au-dessus de tout, y
} 
Pour moi, c'est un endroit où tu peux avoir les meilleures discussions possibles. [...] C'est la règle de base : il ne faut s'offenser de rien... Il ne faut jamais rien prendre au sérieux. La règle officielle c'est : on peut parler de tout. [...] Tu fais une blague un peu raciste, comme tu peux pas le faire en vrai, t'es content, $t^{\prime}$ as fait ta petite blague raciste, tu te sens fort. "

Ce forum est pour Ludovic " un monde parallèle avec tous les rejets de la société, les gens qui sont pas populaires [...] qui parlent pas beaucoup... qui détestent un peu tout. Ils détestent les gens normaux. [...] Ce sont des gens socialement assez nuls, il faut dire. [...] Si vous voulez, on se sent accepté. Personne ne sait qui vous êtes, mais vous êtes un peu chez vous. " L'avantage d'Internet c'est que "personne ne sait qui tu es, ce qui fait que tu peux être n'importe qui... tu peux parler de tout et de n'importe quoi sans risque de jugement. "

II est, sur ses sites, plus facile de se dévoiler et d'y dévoiler son côté obscur. Les relations online donnent un sentiment de sécurité en évitant le danger d'une rencontre offline pouvant virer à la confrontation. Via Internet, la menace du corps, de la présence de l'autre disparaît. Le rapport de force, présent dans tout rapport social, s'efface. L'écran lui-même est une manière de délimiter le pulsionnel du regard et de la voix, dont nous savons à quel point il peut être vécu comme agressif et intrusif. L'écran a finalement (et paradoxalement) le grand avantage de réduire la bande passante. La bande-passante, terme emprunté à l'électronique, désigne la gamme de fréquences qu'un système peut traiter. Réduire la bande passante c'est réduire la transmission des données, l'amplitude des signaux, c'est réduire l'intensité perceptive, ici la riche et complexe expérience phénoménologique. L'écran annule tous les signes de communication dite infra-verbale, expressions du visage, gestes, intonations de la voix, etc. Rencontrer ainsi un autre IRL, in Real Life, c'est rencontrer un visage, un corps, un autre corps que le sien, avec lequel l'adolescent est déjà si souvent en difficulté. C'est recevoir une bande passante beaucoup plus large qui laisse filtrer des signaux qui ne sont pas codés par les interfaces numériques, toute une présence corporelle, et le temps même, la durée de la rencontre car la rencontre sur Internet est a-synchronique.

Ces objets pulsionnels à haut risque que sont la voix et le regard de l'autre sont encadrés (c'est le cas de le dire : l'écran forme un cadre) et même contrôlés par le jeune usager du numérique : rien de plus simple que de couper le son ou d'éteindre son ordinateur en cas de surchauffe. Eteindre c'est finalement effectuer une coupure pulsionnelle. On peut évoquer un parallèle avec le jeu du Fort-Da du petit fils de Freud. Les va-et-vient du sujet et de son smartphone, ou les va-et-vient du sujet avec son ordinateur, sont comme les va-et-vient de l'enfant avec sa bobine: une façon d'apprivoiser l'Autre, ici l'Autre-Internet qui condense toutes les fonctions de l'Autre : demande, désir, jouissance et savoir (nous y reviendrons).

\section{Se soutenir des instances idéales imaginaires}

Un autre usage des interfaces et connexions numériques est d'y sustenter ses images du moi.

Karine, jeune femme de 19 ans en retrait depuis 2 ans, en témoigne. Sur internet, elle s'est créé « un monde ». Elle lit beaucoup de fan fiction, n'arrive pas à s'arrêter de lire des récits de mangas, des épisodes de séries, que des fans écrivent et réécrivent pour poursuivre ou transformer la vie de 
leur(s) personnage(s) fétiche(s). Elle aime les personnages "forts ", "cyniques ", "intelligents", "ironiques ", " beaux ", " au mental d'acier ", " tout ce que je ne suis pas ", "qui font tout ce que je n'ose pas", reconnait-elle. Elle lit sans cesse les "variations", qui réordonnent le récit initial, un personnage mort qui ressuscite, un personnage hétérosexuel qui devient homosexuel, etc. Elle s'est "réfugiée dans un monde de personnages", et s'attache particulièrement à un personnage qui a eu " une enfance douloureuse ", alors qu'elle-même dit si peu de sa propre enfance, personnage qui, évidemment, de ce passé douloureux a su se construire une image valorisante.

Lacan a beaucoup travaillé les instances imaginaires à partir des textes de Freud sur le narcissisme, la pulsion de mort et l'Idéal du Moi (aussi nommé surmoi). Lacan distingue Moi Idéal et Idéal du Moi ${ }^{22}$. Le Moi-Idéal est un idéal de toute puissance basée sur le narcissisme infantile. L'enfant qui se voit dans le miroir se voit autrement qu'il n'est, plus fort qu'il n'est. Il se voit comme son propre idéal. Le narcissisme qui est précisément l'amour porté à sa propre image est malmené par le processus adolescent où le corps est la proie de bouleversements réels, le réel de la puberté non contrôlé par le sujet. Finalement la sexualité arrive trop tôt et le jeune a la tentation de s'en débarrasser ! Le corps possède plutôt l'adolescent que l'adolescent ne possède son corps. Le réconfort du Moi Idéal peut le protéger en contrant l'avènement de réel par un refuge dans l'imaginaire. Un jeune japonais se rêve pilote de génie puisqu'il apprend à piloter via des logiciels de simulation, un jeune homme rencontré à domicile se définit webmaster puisqu'il en a appris les notions online. Mais ni l'un ni l'autre ne confrontent cette image idéalisée en carte d'identité socialement validée. Ni l'un ni l'autre ne songe à transformer ce savoir en un savoir reconnu par l'Autre, en diplôme ou qualification. Ce Moi Idéal que l'adolescent peut se forger via ses usages numériques a le privilège d'échapper aux instances parentales et symboliques. Alors que l'Idéal du Moi, qui est un modèle auquel le sujet cherche à se conformer, a pour objectif de se voir aimable dans le regard de l'Autre, en premier lieu l'autre maternel ou paternel. L'Idéal du Moi est un idéal dans l'Autre, référé au lien à l'Autre et à l'identification d'un trait pris, d'une valeur prise dans cet Autre. Il suppose d'être aliéné à autrui, d'en passer par autrui. L'Idéal du Moi comme identification asymptotique engage le sujet dans une autre relation au temps, nous reviendrons à cette importance de la temporalité dans les usages et connexions numériques. Pour citer Lacan « dans la relation du sujet à l'autre de l'autorité, l'Idéal du Moi, suivant la loi de plaire, mène le sujet à se déplaire au gré du commandement ; le Moi Idéal au risque de déplaire, ne triomphe qu'à plaire en dépit du commandement ${ }^{23}$, citation où l'on saisit les articulations différentes entre le sujet et l'Autre. L'erreur, si on peut parler d'erreur, c'est de viser ce Moi Idéal, et même l'Idéal du Moi, comme des places à prendre, des positions à atteindre, qui paralysent le sujet dans la voie de son désir. L'adolescence est un passage non un état. Quand Moi Idéal et Idéal du Moi se transforment en besoins stables, en zones fixes, ils mettent le sujet en position d'immobilité, du fait du lien entre image et pulsion de mort, même si l'Idéal du moi préserve un lien à l'Autre. L'image survit au vivant, elle le transcende. Prenons l'exemple du stade du miroir, l'enfant est bancal, ses mouvements sont désordonnés, imparfaits, alors que ce qu'il saisit dans le miroir est une image unifiée, idéale. Le sujet peut-il tolérer cette divergence, cet écart entre cette image de lui comme autre qui le ravit à lui-même ? Nous faisons l'hypothèse que cette question est centrale pour les jeunes en retrait.

\footnotetext{
${ }^{22}$ Jacques LACAN distingue ces deux instances dans le Séminaire, livre VIII, Le transfert, Paris, Seuil, 2001.

${ }^{23}$ LACAN, J. 1966, "Remarque sur le rapport de Daniel Lagache : psychanalyse et structure de la personnalité », in Ecrits, Paris, Seuil, pp. 647-684
} 


\section{Accéder au savoir sans passer par un maître}

Internet fournit un réservoir (infini ?) de savoirs, ce qui se dévoile à de nombreuses reprises dans la description des usages des écrans. Michel, jeune homme de 28 ans, qui vient de sortir de 4 ans de retrait, vise grâce à son ordinateur et le réseau Web le savoir encyclopédique d'écrivains ou de réalisateurs. Ludovic y approfondit une passion pour l'histoire, qu'il assouvit à la fois dans des jeux spécifiques, des forums de discussion et des sites spécialisés.

Hanayo, japonaise de 23 ans, en retrait depuis plusieurs mois, témoigne :

"Ces derniers temps, j'ai un grand plaisir à apprendre les langues étrangères en écoutant la radio ou en regardant la télé sur mon ordinateur. Habituellement, j'abandonne vite les choses mais j'écoute la radio tous les jours. J'aimerais pouvoir parler plusieurs langues étrangères un jour. [...] Internet c'est pour l'information. "

Rodolphe, 20 ans, sorti d'un retrait de 4 ans, se sert d'Internet à l'instar de Hanayo dans une volonté d'information et même de formation. Lors de son retrait à domicile, il parvient, malgré un ou deux redoublements, à poursuivre sa scolarité à distance et à apprendre un instrument de musique. "J'ai récupéré des cours, mais aussi des sons, des enregistrements audio [...] aujourd'hui je continue à utiliser internet, je télécharge des partitions pour plusieurs instruments pour mes cours de musique. " Rodolphe a profité de son retrait pour apprendre la musique dont il décide finalement de faire son métier. Dans ces deux exemples d'Hanayo et de Rodolphe - qui ne sont pas les seuls de notre corpus - l'accès au savoir sans passer par l'Autre permet à ces jeunes de redéfinir leurs apprentissages, leurs investissements et leurs motivations professionnelles. Le savoir à disposition sur le Web ne nécessite pas/plus la médiation d'adultes, parents, professeurs ou maîtres. Le savoir n'est plus l'objet d'un Autre. "Auparavant le savoir était un objet qu'il fallait aller chercher dans le champ de l'Autre, il fallait l'extraire de l'Autre par les voies de la séduction, de l'obéissance ou de l'exigence, ce qui nécessitait d'en passer par une stratégie avec le désir de l'Autre. ${ }^{24}$ Le savoir change donc de statut, et surtout semble s'affranchir de médiation humaine, ce qui conduit certains, le philosophe Michel Serres, l'urbaniste Paul Virilio, le linguiste Raffaele Simone, pour en citer quelques-uns, à parler de " révolution civilisationnelle ", "d'apocalypse culturelle » ou de " mutation anthropologique ». Les institutions et la transmission des savoirs sont profondément bouleversées. Le Web, organisation non hiérarchique et hypertextuelle de l'information, brouille la notion d'auteur et remet en cause l'idée d'une intention dans le savoir. L'intention de l'auteur est remplacée par une logique textuelle de contenu. Cette notion d'auteur est aussi profondément remise en question, par la multiplication et l'anonymat des auteurs dans les encyclopédies en ligne comme Wikipédia ou dans la fan fiction qui fait le bonheur de Karine.

Les jeunes connectés à leurs interfaces numériques pratiqueraient non plus une érotique du savoir, qui impliquerait un auteur, un autre par lequel passer pour accéder au savoir, mais une autoérotique du savoir, auto-érotique qui peut s'abstenir d'en passer par autrui, qui peut produire un circuit fermé, court (comme nous l'avons constaté précédemment avec notre jeune japonais pilote d'avion ou notre jeune français webmaster). Cette auto-érotique du savoir est démontrée dans

\footnotetext{
${ }^{24}$ MILLER, Jacques-Alain. En direction de l'adolescence. Intervention de clôture à la $3^{\text {ème }}$ Journée de l'Institut de I'Enfant, Issy-les-Moulineaux, Palais des Congrès, 21 Mars 2015.
} 
l'usage, désormais dénoncé et critiqué, par les moteurs de recherche et les réseaux sociaux d'algorithmes codant les comportements des usagers et ne leur proposant que les connexions qui peuvent leur plaire $!^{25}$ Cette auto-érotique du savoir produirait une certaine dés-idéalisation de l'Autre du savoir, cet Autre dont je n'ai plus besoin pour savoir. Les conséquences de cette désillusion vis-à-vis de l'Autre peuvent se lire dans l'accrochage à un Moi Idéal, plutôt qu'à l'Idéal du Moi. Cet affaiblissement de l'Autre du savoir se repère aussi dans l'utopie anti-autoritaire de l'Internet à ses débuts, utopie toujours agissante chez les libertariens, comme Ludovic, les Anonymous, les cyberactivistes...

\section{S'affranchir de l'espace-temps}

Cet espace particulier de l'Internet s'affranchit des contraintes de l'espace-temps du présent, du « ici et maintenant", du hic et nunc. Ceci nous amène à un dernier point que nous souhaitons développer: les implications spatio-temporelles des usages numériques. C'est très important à noter : les environnements numériques sont des images bi ou tridimensionnelles qui évacuent la $4{ }^{\text {ème }}$ dimension, c'est-à-dire la durée, le temps, en référence justement à l'espace-temps. "L'espace n'y est pas autre chose qu'une image. ${ }^{26}{ }^{26}$ La réalité augmentée désigne d'ailleurs cette superposition d'images à la réalité perçue (comme dans le récent jeu de Pokémon Go). Rappelons-nous que Freud caractérisait le travail de civilisation dans sa très célèbre phrase Wo es War, soll Ich werden, traduite par «là où était le ça, le moi doit advenir. " ${ }^{27}$ Cette phrase lie passé, présent et avenir dans une même formule comme cet autre énoncé freudien qui décrit le travail psychique : « ... passé, présent et futur s'échelonnent au long du fil continu du désir $» .{ }^{28}$ Or, le retrait des jeunes peut apparaitre, surtout quand il s'éternise, comme un temps ni articulé au passé, ni ouvrant sur l'avenir. II n'est pas un temps d'expérience comme l'idée d'un moratoire social, théorisé par Erikson, pourrait le suggérer, il est plutôt un temps d'attente, attente de retrouver le fil du temps, de renouer le fil entre le sujet et son désir. Nous pourrions ainsi définir le retrait des jeunes comme, après le temps de voir - voir son corps transformé par la puberté, voir (et même participer à) la chute des imagos parentaux - et avant le moment de conclure - choisir une identité sexuée, décider d'un métier - un instant de doute, un moment de vérification, une scansion suspensive. L'adolescence est particulièrement liée au temps. Elle est coincée entre l'enfance et la vie adulte. Elle est un retour de l'infantile, qui pour autant n'est pas retour du même. Elle est l'après-coup nécessaire de l'infantile. Elle est aussi le moment de conclure sur le plan de son identification sexuée. Elle est marquée de l'irréversibilité du temps. Winnicott soulignait dans son texte de 1962 que le « seul remède de l'adolescence est le

\footnotetext{
${ }^{25}$ Dans son essai The Filter Bubble ("la bulle filtrante ", Penguin Books, 2011), Eli Pariser, spécialiste du cyberespace, montre comment Google, le moteur de recherche américain, analyse les comportements en ligne et filtre les informations en fonction du profil des usagers. Il affirme que cette sélection permanente, dans tous les domaines - politique, lecture, voyages, culture - fait que Google confine les internautes dans une "bulle cognitive ". Cass R. Sunstein, professeur de droit à Harvard, a été l'un des premiers théoriciens à lancer l'alerte contre le risque de dessèchement intellectuel d'Internet par ces effets de bulle. Cf. un article du Monde en date du 15 septembre 2016 : http://www.lemonde.fr/idees/article/2016/09/15/sur-internet-I-invisible-propagandedes-algorithmes_4998063_3232.html\#u7IOqp08w1TI64pb.99

${ }^{26}$ POTIER, R. 2009, " Au risque du virtuel ? ", Topique, vol 2, n²107, pp.149-162, p.151

${ }^{27}$ Ou dans une traduction plus lacanienne "là où c'était, je dois advenir. "

${ }^{28}$ FREUD, S. (1908) La création littéraire et le rêve éveillé. Traduction de Marie Bonaparte et E. Marty. In Essais de psychanalyse appliquée, Paris, Gallimard, 1971, pp.69-81, Version numérique par J.-M. Tremblay, collection Les classiques des sciences sociales
} 
temps qui passe. ${ }^{29}$ Ce temps est aussi une affaire de grammaire. Si dans les mondes numériques existent les temps du futur et du conditionnel qui sont les temps du virtuel, du potentiel, comme existe le temps présent de l'expérience, on peut se demander ce qu'il en est des autres temps grammaticaux. Qu'en est-il ainsi du futur antérieur? Ce temps de la promesse, ce temps de l'adolescence, le temps par excellence de l'émergence du sujet où le passé prend sens du futur qu'il contient. Le temps immédiat des usages numériques, l'accélération de notre époque moderne, ne privent-ils pas le jeune sujet de nos modernités de ce temps du futur antérieur ? Ce temps qui, après le renoncement, fait la promesse au sujet d'une place dans le monde. "Quand tu auras travaillé, tu auras (peut-être) réussi ", "quand tu auras renoncé à la jouissance, tu y auras droit plus tard", etc. ${ }^{30}$ Winnicott évoque certains sujets non intégrés dans le temps, incapables de mettre en relation le maintenant avec l'ensuite. La difficulté le plus souvent rapportée par les usagers du numérique eux-mêmes est la gestion du temps passé sur Internet, notre étude des temps quotidiens passés devant les écrans en témoigne.

L'adolescence, liée au temps, est indissociable de l'espace. Exister est le problème, la question de l'adolescent, exister, comme l'écrit Lacan en deux mots ex-sister, c'est être " hors de ". L'enjeu pour arriver à exister, pour tout sujet, est de se séparer, de s'exiler de l'infantile, de l'imaginaire oedipien. Cet exil, ce passage " hors de » est fondateur du sujet. Or les usages numériques peuvent inverser un mouvement spatial jusque-là obligé : plutôt que de sortir dans le monde parce que l'adolescent ou le jeune adulte s'intéresse au monde, plutôt que de tendre à ex-sister, le jeune fait entrer le monde chez lui, dans sa chambre, au cœur de la maison familiale, dans un mouvement de repli. L'exil de l'infantile, la séparation d'avec les identifications familiales, en deviennent considérablement plus malaisés.

\section{Conclusion}

Les jeunes en retrait social investissent leurs écrans de façon contrastée. Comme tous les autres usagers numériques, ils ont des motivations diverses pour se connecter: s'informer, jouer, apprendre, participer à des fils de discussion, exprimer ou connaître des avis ou des ressentis, écouter de la musique, regarder des séries, etc. Seul le marketing de soi ne semble pas être leur préoccupation puisqu'ils visent plutôt une forme d'invisibilité sociale. En ce sens, mais en ce sens seulement, leur retrait physique est aussi un retrait numérique. Finalement ces jeunes reclus, ces hikikomori, démontrent qu'Internet n'est pas «Un». Nous confondons souvent la puissance opératoire du réseau, permis par ses outils numériques, avec une unité illusoire. Nous ne pouvons pas identifier Internet à " un " usage, " un " trouble de la conduite ", " un » danger, " une » difficulté, etc. Si nous évoquons le retrait numérique de ces jeunes, c'est à entendre dans une double acceptation : ils sont retirés de la scène Internet comme scène d'exposition de soi, et ils sont retirés de la scène sociale dans des usages numériques chronophages. Car une motivation pour se connecter apparaît plus marquante pour des jeunes claustrés à leur domicile que pour d'autres : passer le temps... "Finalement je passe un peu ma journée dessus ", en arrivent à conclure certains

\footnotetext{
${ }^{29}$ WINNICOTT, DW. L'adolescence (1962) in The Family and individual Development, 1965, Londres, Tavistock. p. 398-399

30 RASSIAL, Jean-Jacques et BENHAÏM, Michèle. 2009, « No future. Grammaire du sujet post-moderne », Cliniques méditerranéennes, $\mathrm{n}^{\circ} 80$, volume $2, \mathrm{p} .287-300$
} 
d'entre eux. Les écrans permettent aux jeunes en retrait d'échapper à l'espace-temps de leur adolescence, qui se caractérise d'être un espace envahi par leur puberté et encombré de leur corps sexué, libidinalisé, et un temps limité, imbriqué, écartelé entre enfance et vie adulte. De toujours l'être humain a voulu / du échapper aux contraintes et aux limites de son corps. De tout temps, l'être humain a cherché à s'échapper, à se libérer des attaches du « ici et maintenant ", à s'affranchir « de l'instantanéité qui caractérise le psychisme animal $\|^{31}$. Le langage, l'imaginaire, la fiction, mais aussi le voyage, les transports, tant de réalisations humaines sont soutenues par ce désir de s'émanciper de limites purement sensibles, strictement corporelles. Les technologies numériques si nous les situons dans cette perspective sont juste un développement complexe et puissant de ce désir profondément humain. Cependant l'espace-temps des écrans comporte une particularité : il est déconnecté du temps commun, des espaces communs. Cet espace-temps des écrans individualisés contribue à protéger les jeunes reclus de la confrontation physique, réelle, corporelle, aux autres. Ils ne sont pas, avec leur corps, en présence des autres, en présence des corps des autres, confrontés aux intentions, aux émotions des autres. Dans ses usages et connexions numériques, le jeune reclus peut s'offrir l'impression d'échapper à des autres plus forts, plus à l'aise (ou qu'il imagine tels), à des maîtres du savoir, au maître du principe de réalité, au maître de la désillusion qui souligne l'écart entre l'idéal de l'image et la réalité en 4 dimensions, il n'échappe cependant pas à ce maître qui lui est si intime : le réel de son propre corps. Ce corps dans le moment de la puberté peut lui apparaitre comme un maître qui s'impose à lui, alors qu'il rêvait, enfant, d'en être maître.

Laissons le mot de la fin à Ludovic, déjà cité, qui avait conclu notre entretien par ce jugement ambigu: s'il n'avait pas eu Internet et les jeux vidéo il serait sorti plus rapidement de son retrait au domicile familial, ou il serait devenu dingue !

\footnotetext{
${ }^{31}$ VUILLEMIN, J. 1948, Essai sur la signification de la mort, Paris, PUF, p. 40.
} 